\title{
NLTE MODEL ATMOSPHERES FOR M DWARFS AND GIANTS
}

\author{
PETER H. HAUSCHILDT \\ Dept. of Physics and Astronomy, Arizona State University, \\ Box 871504, Tempe, AZ 85287-1504 \\ E-Mail: yeti@sara.la.asu.edu \\ FRANCE ALLARD \& DAVID R. ALEXANDER \\ Dept. of Physics, \\ Wichita State University, Wichita, KS 67260-0032 \\ ANDREAS SCHWEITZER \\ Landessterwarte Heidelberg, Königstuhl, D-69117 Heidelberg \\ AND \\ E. BARON \\ Dept. of Physics and Astronomy, University of Oklahoma 440 \\ W. Brooks, Rm 131, Norman, OK 73019-0225
}

\section{Introduction}

The atmospheres of $\mathrm{M}$ stars are dominated by a small number of very strong molecular compounds $\left(\mathrm{H}_{2} \mathrm{O}, \mathrm{TiO}, \mathrm{H}_{2}, \mathrm{CO}, \mathrm{VO}\right)$. Most of the hydrogen is locked in molecular $\mathrm{H}_{2}$, most of the carbon in $\mathrm{CO}$; and $\mathrm{H}_{2} \mathrm{O}$, TiO and $\mathrm{VO}$ opacities define a pseudo-continuum covering the entire flux distribution of these stars. The optical "continuum" is due to $\mathrm{TiO}$ vibrational bands which are often used as temperature indicators for these stars. These may be the depth of the bands relative to the troughs in between them; or the depth of the VO bands; or of the atomic lines relative to the local "continuum"; or even the strength of the infrared water bands; all of these depend on the strength of the $\mathrm{TiO}$ bands and the amount of flux-redistribution to longer wavelengths exerted by them. Departures from LTE of the Ti I atom, and thus the concentration of the important $\mathrm{TiO}$ molecule, could, therefore, have severe and measurable consequences on the atmospheric structure and spectra of these stars.

Due to the very low electron temperatures, the electron density is extremely low in M stars; even lower than in novae and SNe. Collisions with particles other than electrons, e.g., the $\mathrm{H}_{2}$ or helium, are by far not as effective as electron collisions, both because of their smaller cross-sections 


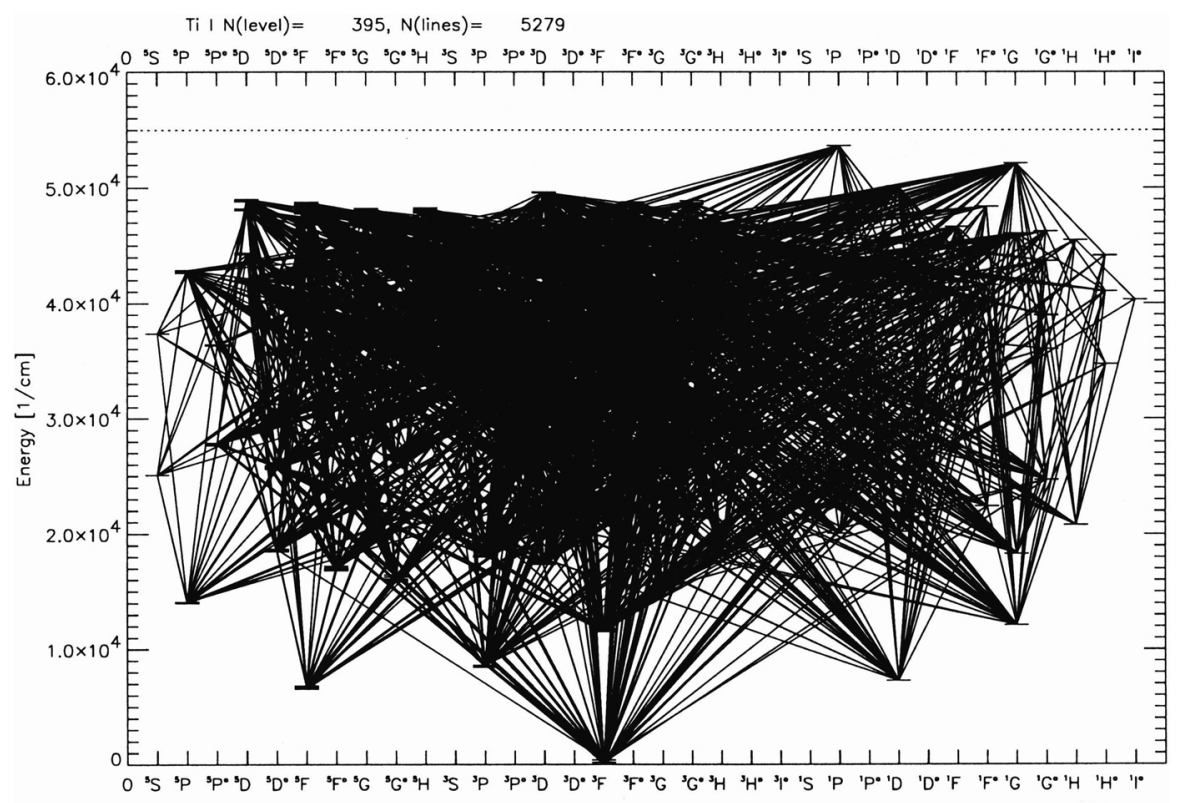

Figure 1. Simplified Grotrian diagram of our Ti I model atom. All 395 levels and 5379 primary (i.e., full NLTE) lines are shown but the 0.8 million secondary (approximate) NLTE lines have been omitted for clarity.

and of their much smaller relative velocities. Therefore, collisional rates which tend to restore LTE, could be very small in cool stars. This in turn could significantly increase the importance of NLTE effects in M stars when compared to, e.g., solar type stars.

In this paper we discuss NLTE effects of Ti I in fully self-consistent models for a few representative $\mathrm{M} /$ Brown dwarf and $M$ giant model atmospheres and spectra.

\section{Methods and Models}

In order to investigate if Ti I NLTE effects are important for the formation of cool star spectra, a full NLTE model calculation is required. This means that the multi-level NLTE rate equations must be solved for a number of species self-consistently simultaneously with the radiative transfer and energy equations, including the effects of line blanketing and of the molecular equation of state. For the purpose of this analysis we use the model code PHOENIX originally developed for the modeling of the expanding atmospheres of novae and supernovae, and adapted to conditions prevailing in cool stars by Allard and Hauschildt (1995, hereafter AH95). PHOENIX 

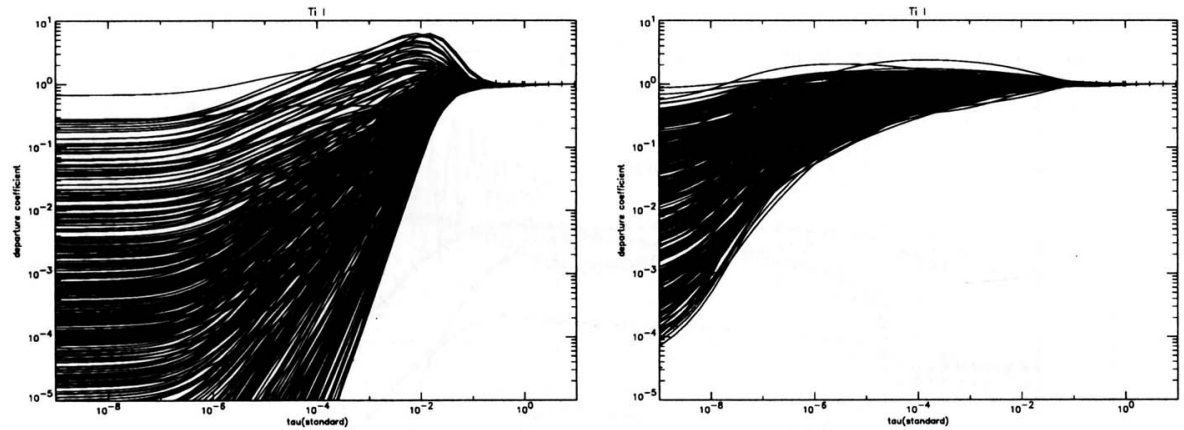

Figure 2. Ti I departure coefficients as function of optical depth for a NLTE model with $T_{\text {eff }}=2700 \mathrm{~K}$ and $\log g=5.0$ (left hand side) and with with $T_{\text {eff }}=4000 \mathrm{~K}$ and $\log g=5.0$ (right hand side).

(version 5.9) uses a spherical radiative transfer for giant models ( $\operatorname{logg}_{i} 3.5$ ), and an equation of state (EOS) recently upgraded from 98 to 195 molecules using the polynomial partition functions by Irwin (1998). For this work, the EOS as been extended to include:

(a) $\mathrm{TiO}^{+}$and $\mathrm{ZrO}^{+}$(Gurvich \& Glushko, 1982).

(b) $\mathrm{H}_{3}^{+}\left(\right.$and $\mathrm{H}_{2}^{+}$) to the EOS using the new $\mathrm{H}_{3}^{+}$partition function by Neale \& Tennyson (1995).

The opacity data base of Phoenix includes assorted $\mathrm{f}-\mathrm{f}$ processes, $\mathrm{H}^{-}$absorption, Collision Induced Absorption (Borysow 1993), JOLA bands for $\mathrm{CaH}, \mathrm{VO}$, and $\mathrm{FeH}$ (AH95), dust absorption (experimental) and a direct line-by-line treatment for all available absorbers (inculding isotopes):

(a) atomic lines from updated Kurucz CD\#1

(b) Jørgensen $\mathrm{TiO} \& \mathrm{CN}$ lines, $\mathrm{H}_{2} \mathrm{O}$ (opacity sampling tables)

(c) Goorvitch $\mathrm{CO}$ lines

(d) the inclusion of the IR roto-vibrational system of $\mathrm{SiO}$ using the detailed line list by Goorvitch.

(e) $\mathrm{H}_{3}^{+}$lines by Neale \& Tennyson (1995)

(f) Kurucz CD\#15 molecular lines

(g) Miller \& Tennyson $\mathrm{H}_{2} \mathrm{O}$ lines for high-res IR spectra

(h) Improved water vapor lines by Viti et al (in preparation) as soon as they become available.

For the strongest ca. $6 \times 10^{6}$ atomic \& molecular lines, we use detailed depth-dependent Voigt profiles with improved damping constant computation by Schweitzer et al (in preparation), and Gaussian profiles for an additional $10 \times 10^{6}$ much weaker lines. In addition, we include ca. 2000 photo-ionization cross sections (Verner \& Yakovlev 1995). Both the NLTE and LTE lines for atoms and molecules are treated with a direct line-by-line (LBL) method. This method is different from the classical opacity sampling 

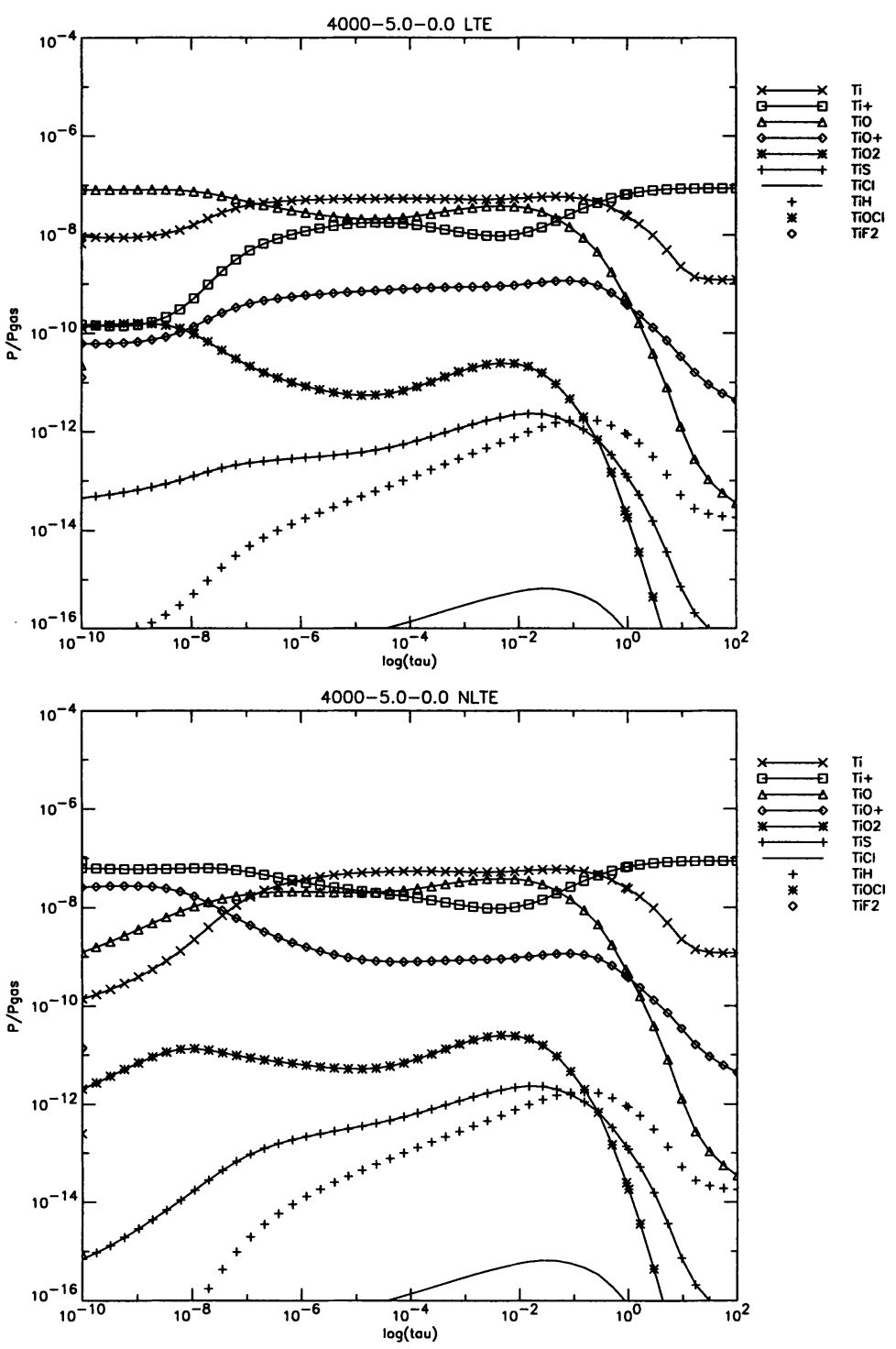

Figure 3. NLTE effects on the $\mathrm{Ti}$ ionization and molecule formation for the $T_{\text {eff }}=4000 \mathrm{~K}, \log g=5.0$ model.

(OS) approach in that we do not use pre-computed tables for the line opacity as a function of temperature using only fixed Gaussian line profiles. The LBL method dynamically selects the relevant lines from the master line lists at the beginning of each iteration and sums the contribution of every line within a search window to compute the total line opacity at $a r$ bitrary wavelength points. The latter is important in NLTE calculations in 

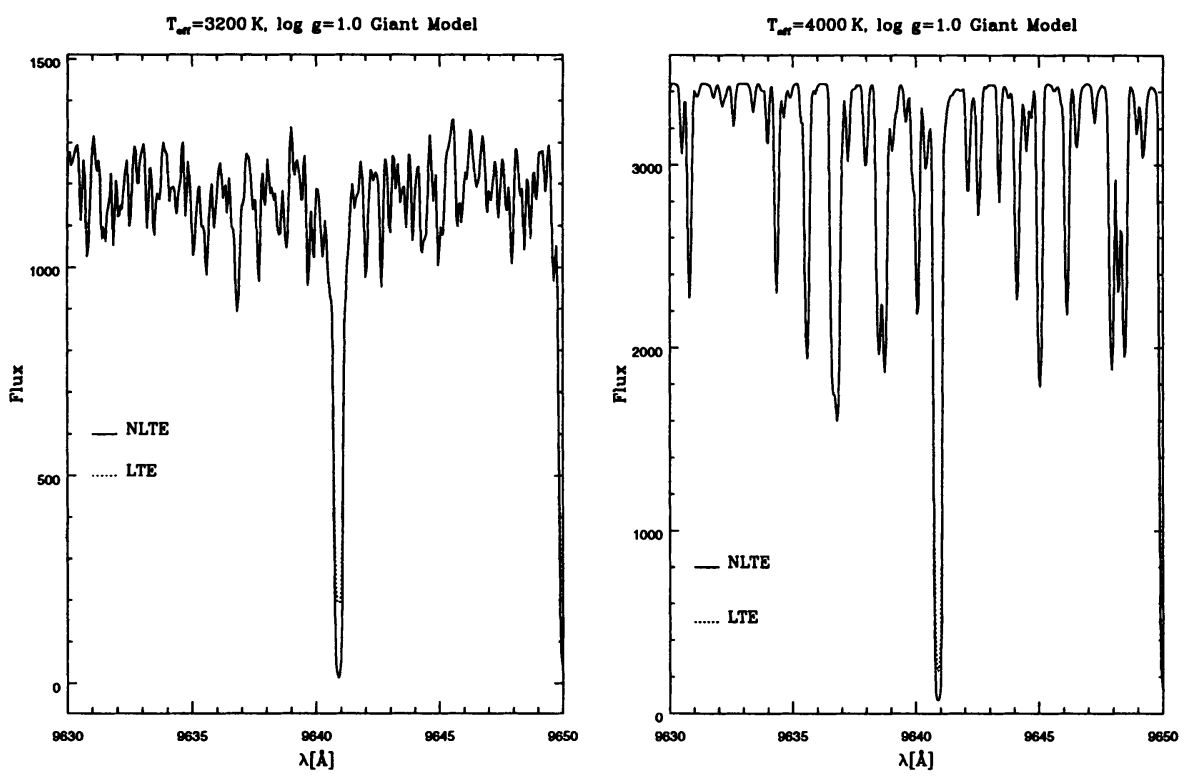

Figure 4. NLTE effects on the Ti I line at $\lambda_{\text {vac }} \approx 9641 \AA$ for two giant models. The model parameters are $T_{\text {eff }}=3200 \mathrm{~K}, \log g=1.0$ for the left hand panel and $T_{\text {eff }}=4000 \mathrm{~K}$, $\log g=1.0$ for the right hand panel. The LTE spectrum uses the same model structure as the NLTE spectrum but with all departure coefficients set to unity.

which the wavelength grid is both irregular and variable (from iteration to iteration due to changes in the physical conditions). The LBL approach also allows detailed and depth dependent line profiles to be used during the iterations. However, in order to make the LBL method computationally feasible, modern numerical techniques, e.g., block algorithms with high data locality, and high-end workstations or supercomputers must be used. In the calculations we present in this paper, we have set the micro-turbulent velocity $\xi$ to $2 \mathrm{~km} \mathrm{~s}^{-1}$. We include LTE lines if they are stronger than a threshold $\Gamma \equiv \chi_{l} / \kappa_{c}=10^{-4}$, where $\chi_{l}$ is the extinction coefficient of the line at the line center and $\kappa_{c}$ is the local b-f absorption coefficient.

PHOENIX is a full multi-level NLTE code, i.e., the NLTE effects are included self-consistently in the model calculations. Hauschildt \& Baron (1995) have extended the numerical method developed by Hauschildt (1993) for NLTE calculations with a very detailed model atom of Fe II. In this section we describe how we apply this technique to add the $\mathrm{Ti} I$ atoms to the list of NLTE species already available in the model calculations. 

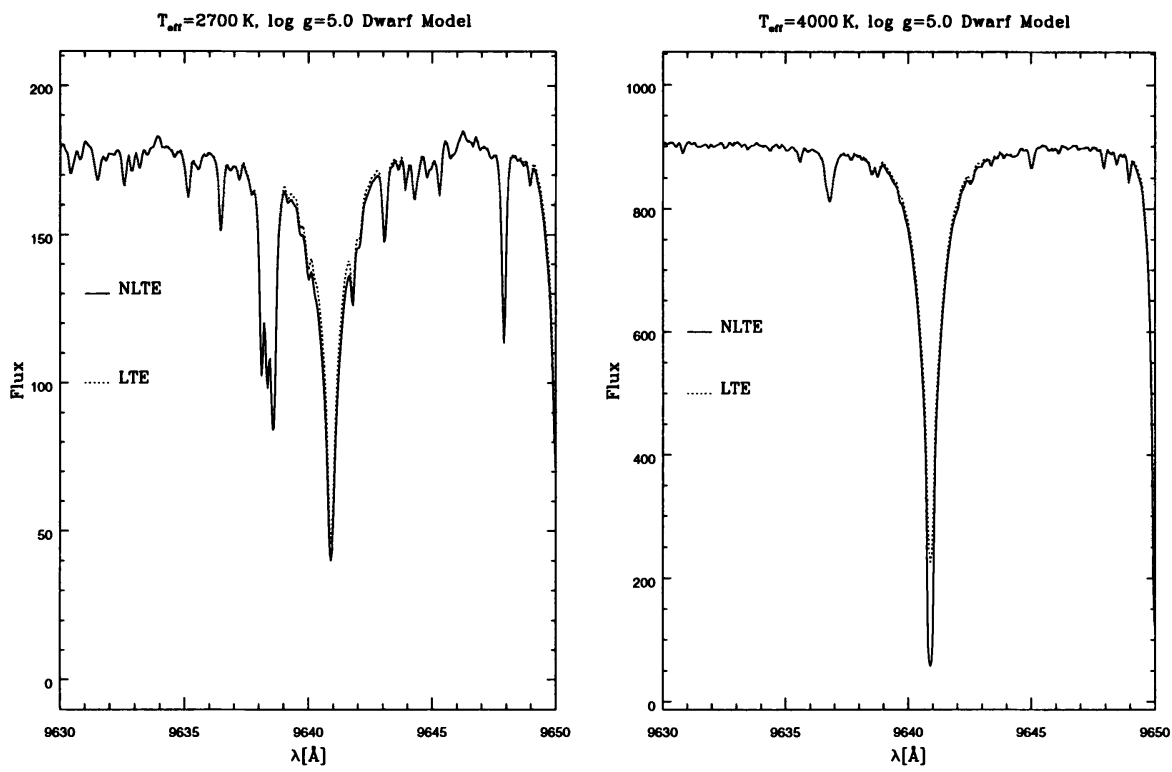

Figure 5. NLTE effects on the Ti I line at $\lambda_{\text {vac }} \approx 9641 \AA$ for two dwarf models. The model parameters are $T_{\text {eff }}=2700 \mathrm{~K}, \log g=5.0$ for the left hand panel and $T_{\text {eff }}=4000 \mathrm{~K}$, $\log g=5.0$ for the right hand panel. The LTE spectrum uses the same model structure as the NLTE spectrum but with all departure coefficients set to unity.

\subsection{THE TI I MODEL ATOM}

To construct the Ti I model atom we have selected the first 34 terms of Ti I. We include all observed levels that have observed b-b transitions with $\log (g f)>-3.0$ as NLTE levels where $g$ is the statistical weight of the lower level and $f$ is the oscillator strength of the transition. This leads to a model atom with 395 levels and 5279 primary transitions treated in detailed NLTE. That is, we solve the complete $b$ - $f \& b$-b radiative transfer and rate equations for all these levels including all radiative rates of the primary lines. A Grotrian diagram of this model atom is shown in Fig. 1. In addition, we treat the opacity and emissivity for the remaining nearly 0.8 million "secondary" $b-b$ transitions in NLTE, if one level of a secondary transition is included in the model. A detailed description of the method is given in Hauschildt \& Baron (1995).

Photo-ionization and collisional rates for Ti I are not yet available. Thus, we have taken the results of the Hartree Slater central field calculations of Reilman \& Manson (1979) to scale the ground state photo-ionization rate and have then used a hydrogenic approximation for the energy variation of the cross-section. Although they are only very rough approximations, the exact values of the b-f cross-sections are not important for the opacities 
themselves which are dominated by known b-b transitions of Ti I and other species. They do, however, have an influence on the actual $b-f$ rates but this remains unimportant for the computational method used in this work.

While collisional rates are important in hotter stellar atmospheres with high electron densities, they remain nearly negligible when compared to the radiative rates for the low electron densities found in cool stars. We have approximated bound-free collisional rates using the semi-enpirical formula of Drawin(1961). The bound-bound collisional rates are approximated by the semi-empirical formula of Allen (1973), while the Van Regemorter's formula (1962) was used for permitted transitions.

A more accurate treatment of this model atom requires the availability of more accurate collisional and photo-ionization rates for Ti I. In the present calculations we have neglected collisions with particles other than electrons because the cross-sections are basically unknown. Additional collisional processes would tend to restore LTE, therefore, the NLTE effects that we obtain in our calculations should be maximized.

\section{NLTE effects on Ti I line profiles in M Star Spectra}

We demonstrate the effects of NLTE on the formation of Ti I lines in Figs. 4 and 5. In general, the NLTE effects are smaller for the cooler models and they are larger for the giants than for the dwarfs at given effective temperature. In the optical spectral region, the changes caused by the Ti I NLTE line formation are very small and would be hard to observe due to the enormous crowding of lines in this spectral region.

In the cooler models, the Ti I lines form deeper in the atmosphere in a region in which the radiation field is nearly Planckian and thus NLTE effects are very small. This is due to the enormous background opacity of $\mathrm{TiO}$ and other molecules. In the outer atmosphere of the cooler models the concentration of $\mathrm{Ti} \mathrm{I}$ is much smaller than that of $\mathrm{TiO}$, thus the effect of the large departures from LTE that we find in these regions on the Ti I line profiles is very small.

In the hotter models, NLTE effects, in particular on the near-IR lines, are much larger. In these models the line forming region of the Ti I lines is inside the region in which the departures from LTE are significant. In addition, the $\mathrm{TiO}$ opacity is relatively smaller than in the cooler models. For the Ti I line at $\lambda_{\text {vac }} \approx 9641 \AA$, NLTE effects make the core of the line deeper than the LTE model predicts. This is due to line scattering which removes photons from the line core and re-distributes them into the line wings. The same effects are present in most $\mathrm{Ti} I$ lines, but there are a few exceptions for which the line is weaker in NLTE than in LTE. Abundance determinations of $\mathrm{Ti}$, or likely all metals, from near-IR or IR lines should 
therefore include NLTE effects wherever possible.

For the model parameters that we have considered so far, the effects of $\mathrm{Ti}$ I NLTE on the TiO bands is very small. This seems to be due to the fact that the line forming region of $\mathrm{TiO}$ (around $\tau_{\mathrm{std}} \approx 10^{-3}$ is dominating the line forming region of the $\mathrm{Ti} I$ lines (due to the larger opacity of the TiO molecule compared to $\mathrm{Ti}$ I). Therefore, the $\mathrm{TiO}$ lines form in a region in which the Ti I atom is basically in LTE and the Ti I NLTE effects that are important at smaller optical depths cannot affect the TiO lines significantly. The situation is different for the $\mathrm{TiO}^{+}$molecule which forms from $\mathrm{Ti}$ II and O I (cf. Fig. 3). This molecule is very sensitive to the Ti I NLTE effects and its lines would be helpful indicators for Ti I NLTE effects.

In further investigations it will be very interesting to look for NLTE effects in the TiO line formation itself. This is certainly feasible with modern numerical techniques once adequate data for $\mathrm{TiO}$ are available.

Acknowledgements: It is a pleasure to thank G. Basri, H. Jones, G. Marcy, S. Starrfield, S. Shore, and S. Viti for stimulating discussions. This work was supported in part by NASA LTSA grants to Arizona State University, by NASA grant NAGW2999 and NSF grant AST-9417242 to University of Oklahoma; as well as NSF and NASA EPSCoR grants to Wichita State University. Some of the calculations presented in this paper were performed at the San Diego Supercomputer Center (SDSC), with support from the National Science Foundation, from the NERSC, and from the U.S. DoE. We thank all these institutions for a generous allocation of computer time.

\section{References}

Allard, F., \& Hauschildt, P. H. 1995, ApJ, 445, 433.

Allen, C. W. 1973, Astrophysical Quantities, Athlone Press, London, third edition.

Drawin, H. W. 1961, Zs. f. Phys., 164, 513.

Gurvich, L., \& Glushko, V. 1982, Thermodinamicheskie Svoistva Individual'nikh Veschev, 4 (Moscow: Sovit Acad. Sci.).

Hauschildt, P. H. 1993, JQSRT, 50, 301.

Hauschildt, P. H., \& Baron, E. 1995, JQSRT, in press.

Irwin, A. W. 1988, A\&A Supp., 74, 145.

Neale, L., \& Tennyson, J. 1995, A\&A, submitted.

Reilman, R. F., \& Manson, S. T. 1979, ApJ Suppl., 40, 815.

Van Regemorter, H. 1962, ApJ, 136, 906.

Verner, D. A., \& Yakovlev, D. G. 1995, A\&AS, 109, 125. 